
\title{
$\begin{array}{ll}\text { Research Square } & \begin{array}{l}\text { Preprints are preliminary reports that have not undergone peer review. } \\ \text { They should not be considered conclusive, used to inform clinical practice, } \\ \text { or referenced by the media as validated information. }\end{array}\end{array}$
}

\section{Effect of "motivational interviewing" and "information, motivation and behavioral skills" on choosing mode of delivery in pregnant women: $A$ randomized controlled trial}

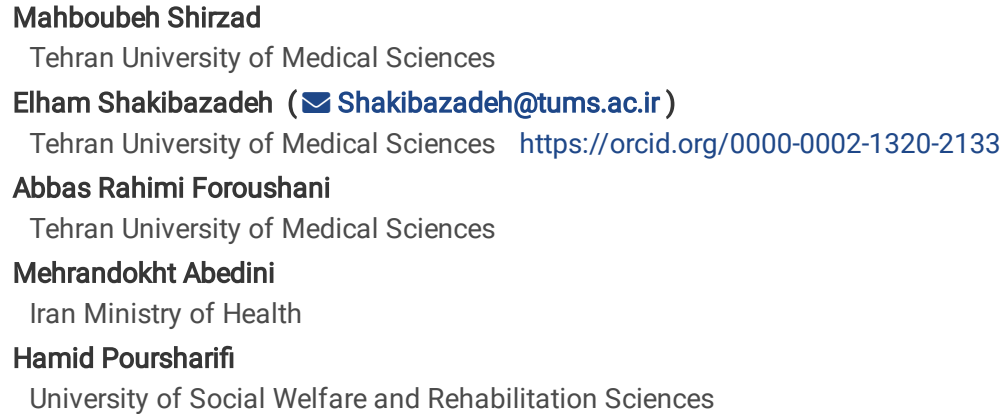




\section{Abstract}

Background: Cesarean section is an important surgical procedure to save mothers and/or babies' lives. Current trends show that the rate of Cesarean section is increasing dramatically over the years. The aims of this study were to compare the effects of "motivational interviewing" and "information, motivation and behavioral skills" model on choosing mode of delivery among pregnant women.

Methods: In a four-armed, parallel-design randomized controlled trial, 120 women were randomly assigned to three brief interventions and one control groups. We recruited pregnant women referring to Ebnesina hospital, Tehran- Iran from 2019-2020. The intervention groups included: 1) motivational interviewing; 2) face-to-face information, motivation and behavioural skills model; and 3) information, motivation and behavioural skills model provided using a mobile application. The control group received usual antenatal care. The inclusion criteria were being literate, gestational age 24 to 32 weeks at recruitment, being able to speak Persian, having no complications in the current pregnancy, having no indications for CS, and having enough time to participate in the intervention sessions. The primary outcome of the study was mode of delivery. The secondary outcomes included women's intentions to undergo any mode of delivery and women's self-efficacy in choosing the mode of delivery. Data were analyzed using descriptive statistics (mean, frequency and standard deviation), inferential statistics including independent $t$-test, paired $t$-test, Chi-squared and One-way ANOVA tests.

Results: Following the interventions, significant differences were found in the mode of delivery $(P<0.05)$ and women's self-efficacy and intention $(P<0.05)$. Women who had used the mobile application had more improvements in the self-efficacy and intention than the face-to-face intervention groups.

Conclusions: Our study showed positive significant effects of various types of brief interventions to reduce unnecessary Cesarean section rate among the participant women. Providing the intervention using mobile application showed even better results. Our findings may contribute to a rise in normal vaginal delivery; and these simple, non-expensive, tailored to women, and culture-oriented brief interventions can be considered as appropriate strategies to reduce Cesarean section rate in local, national, and/or regional levels.

\section{Plain English Summary}

There is a dramatic increase in the rate of Caesarean section in Iran. Several interventions have been implemented to reduce the rate of unnecessary Caesarean section in Iran during last years; however the rate still remains high. In this study, we have conducted a randomized controlled trial with four groups to compare the effects of two models including "motivational interviewing" and "information, motivation and behavioural skills" (face-to-face and via mobile application) on choosing the mode of delivery in 120 pregnant women. These brief pschyo-education interventions were conducted during limited sessions in 24 to 32 gestation weeks for pregnant women. Our findings showed that these brief pchyco-education interventions could motivate women to improve their self-efficacy and intention to choose right mode of delivery. Moreover, it was shown that providing the psycho-education through a mobile phone was more effective than the other two interventions. Delivering these simple, non-expensive, brief educations to pregnant women may reduce the rate of unnecessary Cesarean sections.

\section{Introduction}

Cesarean Section (CS) is an important surgical procedure to save women's and/or babies' lives when medically indicated (1). Despite this fact, this procedure can lead to short- and long-term risks for mother and infant (2-5). World Health Organization (WHO) does not recommend any ideal rate for CS; rather suggests the use of the Robson classification system as a global standard for assessing, monitoring and comparing CS rates within healthcare facilities over time (6).

Iran and Turkey (47.9\% and 47.5\%, respectively) have the highest rates of the CS in Asia (7). Other countries with the highest CS rates are Brazil (55.6\%) and Dominican Republic (56.4\%) in Latin America and the Caribbean (7). In Iran, the rate is even higher in private hospitals (72-89\%) (8-11); and most of the cases have no medical indications in many settings (12).

Studies has identified that unnecessary CSs can create several complications with no benefits to both mother and baby $(2,3,7,13,14)$. CS can be associated with significant short-term risks such as asphyxia. Increasing rates of CS is also associated to increased maternal and perinatal morbidities (15). According to an observational study conducted by WHO in nine Asian countries, women who underwent unplanned CS before or during labor or had assisted (operative) vaginal delivery were more likely to experience morbidity than those who had spontaneous vaginal delivery (16).

Previous studies have showed a list of probable reasons for high rate of CS including several economic, organizational, social and cultural issues (17, 18). The underlying factors of continuing high rates of CS have been studied in a mixed method review in Iran (19). This review reported that preferences for CS were often associated with all 'women's factors', 'health professional factors', and 'health organization, facility, or system factors'(19). The review showed that most of the women had fear of pain during labor and childbirth (20-22), had concerns about genital modifications after vaginal delivery(20, 22-24), believed that CS was safer for baby(25-28), and believed that CS was convenient for women and their families (27). Other studies show that women can play a major role in decision-making process about their birth $(22,29,30)$.

In view of this unprecedented rise of CS in Iran, different interventions have been designed to reduce this trend (31-34). In Iran, many efforts have been made during last years to reduce CS rate. For instance, in 2014, Iran has been conducted a "health sector evolution policy" to improve public health; and increasing the rate of normal vaginal delivery (NVD) was one the most important objectives of this policy (35). Several strategies have been conducted through this policy such as freeing NVDs in all public hospitals, developing mother-friendly hospitals, developing standard protocols of birth and preparation classes for women, improving privacy and infrastructure of labor, promoting standards in birth facilities, promoting water birth, determining financial incentives to doctors 
to encourage them to do NVDs in public hospitals (36). There was a reduction in CS rate after implementing the policy; however the rate is still significantly higher than the rate recommended by WHO (36).

The WHO recommended that non-clinical interventions can be effective to reduce unnecessary CSs. The recommendations are grouped according to the target of intervention: (a) interventions targeted at women, (b) interventions targeted at health-care professionals; and (c) interventions targeted at health organizations, facilities or systems (37). According to this guideline, an important target for interventions is women. Previous experiences also makes it explicit that, in order to further reduce the rate of CS, it is necessary not only to address health system, health facility, and health professional factors, but also change women's choice behaviors (36).

Implementing psycho-education interventions for women has been recommended by WHO in order to reduce unnecessary CS (38, 39). A Cochrane systematic review conducted by Chen et al (2018) on non-clinical interventions for reducing unnecessary CS reported that psycho-education interventions were effective in reducing unnecessary CSs (40). The educational interventions included psycho-education on fear of childbirth (41), intensive group therapy (cognitive behavioral therapy and childbirth psycho-therapy) (42), psycho-education by telephone (43), role-play education versus standard education using lectures (44), and nurse-led applied relaxation training program (41).

Motivational interviewing $(\mathrm{Ml})$ is a patient-centered counseling approach that has been proved to be influential in choosing suitable health behaviors (45); and is specifically aimed to improve motivation to change among individuals not ready to change unhealthy behavior (46). Research on MI has demonstrated positive effects of helping patients clarify goals, explore obstacles to treatment, and make commitments to change (46).

In Information-Motivation-Behavioural skills (IMB) model, individuals should be informed, motivated and behaviorally skilled to change behaviour (47). The IMB model is a generalizable, and simple model to guide thinking about complex health behaviours. The IMB constructs, and how they pertain to patient adherence, are outlined as follows: 1) Information is the basic knowledge about a medical condition that might include how the disease develops, its expected course and effective strategies for its management; 2) Motivation encompasses personal attitudes towards the adherence behaviour, perceived social support for such behaviour, and the patients' subjective norm or perception of how others with this medical condition might behave; and 3) Behavioural skills include ensuring that the patient has the specific behavioural tools or strategies necessary to perform the adherence behaviour such as enlisting social support and other self-regulation strategies. The IMB model has been widely used in changing behavior for health problems (48-51).

In the modern age of information and communication, mobile applications play an important role in delivering educational contents nowadays. Educational interventions should be delivered at any time to anyone with extra support upon to request wherever and whenever it is needed (52); and mobile applications can easily make this access to people. This route provides simple, user friendly, downloadable, and non-expensive interventions to various ranges of individuals. Motivational messages, monitoring, and behaviour change tools can be modified for delivery via mobile phones (53). This type of interventions have been reported to be effective in several behavior change studies including smoking cessation (54), adherence to prescribed medication (55), blood pressure management; and delivering interventions(56) .

The aim of this study was to compare the effect of "motivational interviewing" and "information, motivation and behavioral skills" with face-to-face and mobile application in brief psychological interventions on women's self-efficacy, intention to choose mode of delivery, and the mode of delivery among pregnant women.

\section{Methods}

\section{Trial design}

This was a four-armed randomized controlled, parallel-design trial.

\section{Participants}

We recruited 120 pregnant women referring to Ebnesina private hospital, Tehran- Iran from 2019-2020. The inclusion criteria were being literate, being in gestational age 24 to 32 weeks at recruitment, being able to speak Persian, having no complications in current pregnancy, having no indications for CS, and having enough time to participate in the intervention's sessions. We excluded women who showed complications during the study, had preterm labour, missed at least one session of the interventions and were reluctant to continue to participate in this study (57).

\section{Interventions}

We had three intervention and one control groups. Educational contents were developed using findings of a qualitative evidence synthesis and a quantitative systematic review and meta- analysis (19) and a qualitative study conducted in Iran (35).

The interventions included: 1) motivational interviewing (IM); 2) information, motivation and behavioral skill model through face-to-face approach (IMB); and 3) information, motivation and behavioral skill model through Mobile App (IMB-App). We designed three-session brief interventions and scheduled them within the participants' time and prenatal care visit appointments for MI and IMB groups. All MI and IMB sessions were held by a midwife and a behavioral specialist that were trained by a health psychologist (H. P.). The IMB-App was designed in three sessions similar to the IMB group. The first session was accessible to the participants at the time of recruitment. Other session was accessible after the participants practiced the previous session. There was no time scheduling in the IMB-App group. The control group received usual antenatal care in the clinic.

\section{Motivational interviewing (MI)}


In this intervention group, pregnant women were interviewed face-to-face during three 45-60 minutes sessions. MI techniques were provided to the participants. $\mathrm{MI}$ is a directive, client-centered counseling style for eliciting behavior change by helping clients to explore and resolve ambivalence. It is most centrally defined not by technique but by its spirit as a facilitative style for interpersonal relationship. MI is a relatively simple, transparent and supportive talk therapy based on the principles of cognitive-behavior therapy. In this intervention group, we helped women to explore and resolve ambivalence about mode of delivery and build their intrinsic motivation. We did not force women to choose a specific mode of delivery. We asked open-ended questions (for example "Tell me what you think about CS?" or "What do encourage you to choose this type of delivery (CS or NVD)?"). Open-ended questions could help us to understand how they were thinking about mode of delivery. Affirming is one of the fundamental MI skills. We used it to support engagement, encourage the women to further explore change processes and build confidence. We found an opinion the client was making or a strength she noticed and reflected it back to her (for example" So how did you manage to control your fear after attending our training sessions?"). We used reflective listening. It is a simple method to reduce resistance in $\mathrm{Ml}$ and the last step of this technique is to summarize what the pregnant women have said (Table 1).

\section{Information, motivation and behavioral skill model through face-to-face approach (IMB)}

The model's strategies were presented to the pregnant women, in three 45-60 minutes sessions. Women have received information and behavioral skills related to the choice of mode of delivery as well as internal and external motivational factors related to the choice of delivery. The strategy included three sessions as follow: Session 1) information: the intervention began with information on prevalence of CS and CS-related complications in women; and outcome of unnecessary CS; Session 2) motivation: the interventionist performed this technique to motivate pregnant women; providing personal feedback, asking open ended questions, affirming desirable behavior, reflective listening, working at the women's pace and negotiating goals that was realistic and attainable; Session 3) behavioral skills: women were given behavioural skills training on how to control the obstacle of NVD. To build skills for choosing the mode of delivery, training was given on how reduce these barriers. In the last session, final assessment was also conducted (Table 1).

\section{Information, motivation and behavioral skill model through Mobile App (IMB-App)}

The mobile application (M-health) had designed based on IMB model (flowchart 1 and appendix 1-9). The content was the same as the IMB intervention that was presented in three similar sessions in the Mobile Application. The software was installed on mobile phones of participants in this group; and its operation was taught individually to them. Women worked with the application in presence of one of the researchers, and any existing problems were resolved. The strategies foreseen for adherence improvement included reminders set at defined intervals in the form of pop-up messages. In order to monitor adherence, the data collected on the server were used. In addition to the application, a server was designed in which the users' activities were collected. Items such as the duration of application usage, the sections were used by the user (in addition to registering their time and duration) (appendix 10), etc. was registered. Every time the user's mobile was connected to the Internet, the data was uploaded and saved on to the server. These data could be used as a proxy of adherence to the intervention.

Table 1: Interventions by four study groups

\begin{tabular}{|c|c|c|c|c|}
\hline $\begin{array}{l}\text { Training } \\
\text { session }\end{array}$ & $\mathrm{MI}^{1}$ group & IMB $^{2}$ group & $\begin{array}{l}\text { IMB- } \\
\text { App } \\
\text { group }\end{array}$ & \begin{tabular}{|l|l|l} 
Control group \\
\end{tabular} \\
\hline $\begin{array}{l}\text { First } \\
\text { session }\end{array}$ & $\begin{array}{l}\text { Familiarization: Introduction, norms and group process, } \\
\text { facilitating philosophy, practice of freedom, practice of } \\
\text { dimensions of effect of behavior, practice of change of } \\
\text { assessment change. } \\
\begin{array}{l}\text { Emotions: Identifying emotions, exercising and completing } \\
\text { practice dimensions of influence with emotional dimensions and } \\
\text { homework }\end{array}\end{array}$ & $\begin{array}{l}\text { Information: introducing and explaining the program of norms } \\
\text { and standards of the group, asking questions to start the } \\
\text { discussion about CS and NVD, rate of CS, prevalence of CS, } \\
\text { risk factors for CS, consequences of unnecessary CS }\end{array}$ & $\begin{array}{l}\text { Same } \\
\text { as } \\
\text { IMB } \\
\text { group }\end{array}$ & $\begin{array}{l}\text { Usual } \\
\text { antenatal } \\
\text { care }\end{array}$ \\
\hline $\begin{array}{l}\text { Second } \\
\text { session }\end{array}$ & $\begin{array}{l}\text { Pros and cons of the CS and NVD: brainstorming of short-term } \\
\text { and long-term pros and cons of the CS, completing table of the } \\
\text { pros and cons of the CS and NVD, describe and practice about } \\
\text { the NVD }\end{array}$ & $\begin{array}{l}\text { Motivation: asking open ended questions about unnecessary } \\
\text { CS, providing personal feedback, reasons for needing to } \\
\text { change current behaviour, feedback and affirmation, affirming } \\
\text { desirable behavior, reflective listening, working at the } \\
\text { women's pace and negotiating goals that was realistic and } \\
\text { attainable and empowerment to change behaviour, } \\
\text { summarizing, discussing. }\end{array}$ & $\begin{array}{l}\text { Same } \\
\text { as } \\
\text { IMB } \\
\text { group }\end{array}$ & $\begin{array}{l}\text { Usual } \\
\text { antenatal } \\
\text { care }\end{array}$ \\
\hline $\begin{array}{l}\text { Final } \\
\text { session }\end{array}$ & $\begin{array}{l}\text { Values, Perspective and Final Assessment: Defining values } \\
\text { (what is the important thing for women?), identification and } \\
\text { prioritization of first class values, adaptation of value and mode } \\
\text { of delivery, summarizing and summarizing practice of previous } \\
\text { sessions in perspective practice training be prepared to start a } \\
\text { behaviour change program (decision about NVD). Final } \\
\text { measurement. }\end{array}$ & $\begin{array}{l}\text { Behavioural Skills: how they should cope with NVD, and how } \\
\text { they can control their problems with NVD and conclusion. Final } \\
\text { measurement. }\end{array}$ & $\begin{array}{l}\text { Same } \\
\text { as } \\
\text { IMB } \\
\text { group }\end{array}$ & $\begin{array}{l}\text { Usual } \\
\text { antenatal } \\
\text { care } \\
\text { Final } \\
\text { measurement }\end{array}$ \\
\hline
\end{tabular}

1 Motivational Interviewing; ${ }^{2}$ Information, Motivation, Behavioral Skill Model; ${ }^{3}$ Information, Motivation, Behavioral Skills Model through mobile application

\section{Outcomes}

Primary outcome of the study was the mode of delivery. The secondary outcomes were women's intention to undergo CS and women's self-efficacy. The outcomes questionnaires along with the demographic and obstetrics characteristics were administered after the recruitment of the participants. The outcomes were measured once again at the end of last sessions.

Self-efficacy is a belief that individuals have about themselves' ability to cope with stressful situations and implement necessary actions successfully. There is a known relationship between fear of childbirth, self-efficacy and NVD; if a pregnant woman believes that she cannot deal with labor situation, her fear and anxiety will lead her to choose CS without medical indications (58). 
Regarding intention to choose a mode of delivery, we asked women about their intention about their mode of delivery at baseline and at the end of last session.

\section{Sample size}

The sample size was calculated to be 120 pregnant women (30 women in each group) with a power of $80 \%$ to detect a minimum difference of at least $20 \%$. Considering that the ratio of CS in the control group is $50 \%$, which in previous reports was $47.9 \%$ (116), the number of samples in each group was determined with $95 \%$ confidence, $80 \%$ test power. We replaced other participants with ones who were no longer participated in the study for any reason.

\section{Randomization}

The participants were randomly assigned to four groups after the initial assessment and upon completing the baseline measurement. We recruited the participants based on registration order of women with clinic, and no other factor contributed to the participants' order on the list. Each participant on the list assigned a consecutive research identification number according to the order by which they were registered with the clinic. The first participant on the list was randomly assigned to the MI intervention group, and the next two participants were assigned to the IMB and IMB-App interventions, respectively. The forth group of participants was assigned to the control group.

\section{Blinding}

To avoid bias in the outcome assessment, researchers concerned with data collection and/or preparation were blind to the allocation of the participants.

\section{Measures}

The questionnaires contained the women's demographic information, self-efficacy to choose mode of delivery, and intention behavior. The validity and reliability of the questionnaires have been examined and verified $(59,60)$. The demographic section included items on age, income, educational level (pregnant women and their partners), employment status (pregnant women and their partners), number of births, number of pregnancies, current gestational age (at recruitment), number of live children, history of infertility, history of illness, date of birth, participating in birth classes, and preferred mode of delivery. The questionnaire also consisted of 17 items about self-efficacy and two items about intention to choose mode of delivery. The Cronbach's coefficient alpha was calculated to test the reliability; and exploratory factor analysis was conducted to examine construct validity of the Persian version of the questionnaires.

Follow-up: We followed the participants until time of delivery to determine final mode of delivery. The pregnant women were participated in this study after ensuring that they had study criteria. They completed informed consent forms. In the first visit, the baseline data questionnaire was completed by the researcher. During the second visit, the application was installed on cell phones of the IMB-App group and its operation was taught to them.

Statistical methods: Data were analyzed using SPSS 16 using descriptive statistics (mean, frequency and standard deviation), inferential statistics including independent-t-test, Paired-t-test, Chi-squared test, and analysis of variance (ANOVA) modeling to examine the factors affecting the women's choice on mode of delivery in order to examine the simultaneous effect of variables on the chances of choosing CS. The significance level of the tests was less than 0.05 .

Role of the funding source: The roles of the funders were to monitor the corresponding research planning and progression.

\section{Results}

Demographic characteristics of the participants are shown in Table 1. Most of the participants (57.5\%) were 18-30 years old. Most of the women (57.5\%) were nulliparous and were employed (70.0\%). Our findings showed that there were no significant differences between the groups at baseline measurement in terms of age, educational level of pregnant women and their partners, income, occupation of pregnant woman, and source of information about mode of delivery.

Table 1: Demographic characteristics of the pregnant women in four groups 


\begin{tabular}{|c|c|c|c|c|c|c|c|c|c|c|c|c|}
\hline \multirow{2}{*}{\multicolumn{2}{|c|}{ Demographic characteristics }} & \multicolumn{2}{|c|}{$\mathrm{MI}^{1}$} & \multicolumn{2}{|c|}{$\mathrm{IMB}^{2}$} & \multicolumn{2}{|c|}{ IMB-App $^{3}$} & \multicolumn{2}{|c|}{$\begin{array}{l}\text { Control } \\
\text { Group }\end{array}$} & \multicolumn{2}{|c|}{ Total } & \multirow{3}{*}{$\begin{array}{c}\mathbf{X}^{2} \\
\text { P-value } \\
0178\end{array}$} \\
\hline & & $\mathrm{N}$ & $\%$ & $\mathrm{~N}$ & $\%$ & $\mathrm{~N}$ & $\%$ & & & $\mathrm{~N}$ & $\%$ & \\
\hline \multirow[t]{2}{*}{ Women's age } & $18-30$ years & 18 & 60.0 & 16 & 53.3 & 13 & 43.3 & 10 & 33.3 & 56 & 57.5 & \\
\hline & $>30$ years & 12 & 40 & 14 & 46.7 & 17 & 56.7 & 20 & 66.7 & 63 & 52.5 & \\
\hline \multirow[t]{2}{*}{ Parity } & Nulliparous & 14 & 46.7 & 20 & 66.7 & 21 & 70.0 & 14 & 46.7 & 69 & 57.5 & 0.242 \\
\hline & Multiparous & 16 & 53.3 & 10 & 33.3 & 9 & 30.0 & 16 & 53.3 & 51 & 42.5 & \\
\hline \multirow[t]{3}{*}{ Number of previous deliveries } & 1 & 14 & 46.7 & 23 & 76.7 & 22 & 73.3 & 15 & 50.0 & 74 & $\begin{array}{ll}61.7 \\
3.7\end{array}$ & 0.106 \\
\hline & 2 & 10 & 33.3 & 6 & 20.0 & 5 & 16.7 & 11 & 36.7 & 32 & 26.7 & \\
\hline & $2<$ & 6 & 20.0 & 1 & 3.3 & 3 & 10.0 & 4 & 13.3 & 14 & 11.7 & \\
\hline \multirow[t]{3}{*}{ Number of children } & 0 & 12 & 40.0 & 18 & 60.0 & 24 & 80.0 & 16 & 53.3 & 70 & 57.3 & 0.086 \\
\hline & 1 & 12 & 40.0 & 9 & 30.0 & 4 & 13.3 & 11 & 36.7 & 36 & 30.0 & \\
\hline & $1<$ & 6 & 20.0 & 3 & 10.0 & 2 & 6.70 & 3 & 10.0 & 14 & 11.7 & \\
\hline \multirow[t]{4}{*}{\begin{tabular}{|l} 
Ethnicity \\
\end{tabular}} & Azari & 12 & 40.0 & 11 & 36.7 & 21 & 40.0 & 11 & 36.7 & 46 & 38.3 & 0.267 \\
\hline & Fars & 5 & 16.7 & 13 & 43.3 & 7 & 46.7 & 10 & 33.3 & 42 & 35.0 & \\
\hline & Kord & 7 & 23.3 & 4 & 13.3 & 2 & 10.0 & 5 & 16.7 & 19 & 15.8 & \\
\hline & Others & 6 & 20.0 & 2 & 6.7 & 1 & 3.3 & 4 & 13.3 & 13 & 10.8 & \\
\hline \multirow[t]{2}{*}{ Income per month } & $<50$ million Rial & 20 & 66.7 & 19 & 63.3 & 17 & 56.7 & 19 & 63.3 & 75 & 62.5 & 0.879 \\
\hline & 50-100 million Rial & 10 & 33.3 & 11 & 36.7 & 13 & 43.3 & 11 & 36.7 & 45 & 37.5 & \\
\hline \multirow[t]{3}{*}{ Women's education level } & $<12$ years & 5 & 16.7 & 2 & 6.7 & 1 & 3.3 & 2 & 6.7 & 10 & 8.3 & 0.095 \\
\hline & High school diploma & 7 & 23.3 & 1 & 3.3 & 8 & 26.7 & 6 & 20.0 & 22 & 18.3 & \\
\hline & college or university & 18 & 60.0 & 27 & 90.0 & 21 & 70.0 & 22 & 73.3 & 88 & 73.3 & \\
\hline \multirow[t]{3}{*}{ Spouse's education level } & $<12$ year & 6 & 20.0 & 2 & 6.7 & 3 & 10.3 & 2 & 6.7 & 13 & 10.8 & 0.370 \\
\hline & High school diploma & 7 & 23.3 & 4 & 13.3 & 6 & 20.7 & 9 & 30.0 & 27 & 22.5 & \\
\hline & College or university & 17 & 56.7 & 24 & 80.0 & 20 & 69.0 & 19 & 63.3 & 80 & 66.7 & \\
\hline \multirow[t]{2}{*}{ Women's job status } & Employed & 19 & 63.3 & 21 & 70.0 & 20 & 66.7 & 24 & 80.0 & 84 & 70.0 & 0.528 \\
\hline & Housewife & 11 & 36.7 & 9 & 30.0 & 10 & 33.3 & 6 & 20.0 & 36 & 30.0 & \\
\hline \multirow[t]{2}{*}{ Prenatal education } & No education & 20 & 66.7 & 17 & 56.7 & 21 & 70.0 & 16 & 53.3 & 74 & 61.7 & 0.760 \\
\hline & Yes & 10 & 33.3 & 13 & 43.4 & 9 & 30.0 & 14 & 46.6 & 46 & 38.4 & \\
\hline \multirow[t]{5}{*}{ Preferred method of receiving education } & By phone & 7 & 23.3 & 5 & 16.7 & 10 & 33.3 & 7 & 23.3 & 29 & 24.2 & \multirow[t]{5}{*}{0.520} \\
\hline & Face-to-face & 4 & 13.3 & 3 & 10.0 & 4 & 13.3 & 6 & 20.0 & 17 & 14.2 & \\
\hline & Film & 5 & 16.7 & 7 & 23.3 & 8 & 26.7 & 2 & 6.7 & 22 & 18.3 & \\
\hline & Book & 4 & 13.3 & 2 & 6.7 & 3 & 10 & 4 & 13.3 & 13 & 10.8 & \\
\hline & Group classes & 10 & 33.3 & 13 & 43.3 & 5 & 16.7 & 11 & 36.7 & 39 & 32.5 & \\
\hline
\end{tabular}

${ }^{1}$ Motivational Interviewing; ${ }^{2}$ Information, Motivational and Behavioral skills model; ${ }^{3}$ Information, Motivational and Behavioral skills model through mobile application

Mean scores of self-efficacy and intention are shown in Table 2. The results of the paired samples $t$-test showed that there was a significant increase in the self-efficacy and intention scores of the pregnant women in all intervention groups after the interventions. Findings showed that the psycho-education interventions had significant effect on the mean score of preferred mode of delivery among pregnant women in all intervention groups.

Table 2: Comparison of the mean scores, before and after the psycho-education interventions according to the study groups, using oneway ANOVA

\begin{tabular}{|c|c|c|c|c|c|c|c|c|c|c|}
\hline \multirow[t]{3}{*}{ Variables } & $\mathrm{MI}^{1}$ group & & \multirow{3}{*}{$\begin{array}{c}\text { p- } \\
\text { value }\end{array}$} & IMB $^{2}$ group & & \multirow{3}{*}{$\begin{array}{c}\text { p- } \\
\text { value }\end{array}$} & \multicolumn{2}{|c|}{ IMB-App group } & \multirow{3}{*}{$\begin{array}{l}\text { p- } \\
\text { value }\end{array}$} & Contro \\
\hline & Before & fter & & Before & After & & Before & After & & Before \\
\hline & Mean \pm SD & Mean \pm SD & & Mean \pm SD & Mean \pm SD & & Mean \pm SD & $\mathrm{SD}$ & & $1 \pm \mathrm{SD}$ \\
\hline se & 66.1 & 80. & O & & 103 & $<$ & 77 & .71 & 0 & $7 \pm 40.82$ \\
\hline Intention & $1.17 \pm 0.379$ & $1.57 \pm 0.504$ & 0.000 & $1.33 \pm 0.479$ & $1.73 \pm 0.450$ & 0.000 & $1.10 \pm 0.305$ & $1.70 \pm 0.466$ & 0.000 & $1.30 \pm 0.466$ \\
\hline
\end{tabular}

Mean values were significantly different from those before the intervention (paired-samples t-tests): $*$ P $<0.05$.; Motivational Interviewing Group; ${ }^{2}$ Information, Motivation and Behavioural Modal Group; ${ }^{3}$ Information, Motivation and Behavioural Model through mobile application Group

Based on the paired-comparison t-test results, the mean self-efficacy score of pregnant women in the IMB-App group was $77.1 \pm 38.6$ before the intervention and $99.7 \pm 30.7$ after the intervention. In the IMB group, mean self-efficacy was $86.8 \pm 24.5$ before and $103.2 \pm 24.9$ after the intervention; and in the MI group, it was $66.1 \pm 18.2$ before and $80.5 \pm 22.7$ after the intervention. Therefore, significant differences were observed between the four groups ( $p$.value $<0.001)$ after the interventions. There were no significant differences before and after the interventions in the control group (p.value $<0.47)$. Women's self-efficacy had been increased more in the IMB-APP group than the IMB and MI groups.

An independent-samples t-test was conducted to compare self-efficacy in CS and NVD delivery before and after the interventions. There was a significant difference in the scores for CS $(66.30 \pm 04.71)$ and NVD $(89.04 \pm 34.28)$ delivery; $t=2.978, p=0.004$ before and a significant difference in the scores for CS $(86.41 \pm 30.62)$ and NVD $(101.09 \pm 27.53)$ delivery; $t=2.637, p=0.009$ after the intervention. These results suggested that self-efficacy really did have an effect on mode of delivery choice (Table 3$)$.

Table 3: Comparison of self-efficacy in women undergone CS or NVD 


\begin{tabular}{|l|c|c|}
\hline \multirow{2}{*}{ Self-efficacy } & Before intervention & After intervention \\
\cline { 2 - 3 } Mode of delivery & Mean \pm SD & Mean \pm SD \\
\hline NVD $^{1}$ & $89.04 \pm 34.28$ & $101.09 \pm 27.53$ \\
\hline $\mathrm{CS}^{2}$ & $66.30 \pm 04.71$ & $86.41 \pm 30.62$ \\
\hline Result & $\mathrm{t}=2.978, \mathrm{p}=0.004$ & $\mathrm{t}=2.637, \mathrm{p}=0.009$ \\
\hline
\end{tabular}

Mean values were significantly different from those before the intervention (independent sample t-tests): $* \mathrm{P}<0.05 ;{ }^{1}$ Normal Vaginal Delivery; ${ }^{2}$ Cesarean Section

Women's intention to undergo CS decreased in the intervention groups after the interventions (P-value $<0.001)$; and this decrease occurred more among women in the IMB-App group than the other intervention groups (Table 4).

Table 4: Comparison of women's intention on mode of delivery at baseline and after the intervention in each group after the interventions

\begin{tabular}{|c|c|c|c|c|c|c|c|c|c|c|c|c|c|c|c|c|}
\hline \multirow[t]{3}{*}{ Variables } & \multicolumn{4}{|c|}{$\mathrm{MI}^{1}$ group } & \multicolumn{4}{|c|}{ IMB $^{2}$ group } & \multicolumn{4}{|c|}{ IMB-App ${ }^{3}$ group } & \multicolumn{4}{|c|}{ Control group } \\
\hline & \multicolumn{2}{|c|}{ Before } & \multicolumn{2}{|c|}{ After } & \multicolumn{2}{|c|}{ Before } & \multicolumn{2}{|c|}{ After } & \multicolumn{2}{|c|}{ Before } & \multicolumn{2}{|c|}{ After } & \multicolumn{2}{|c|}{ Before } & \multicolumn{2}{|c|}{ After } \\
\hline & $\mathrm{N}$ & $\%$ & $\mathrm{~N}$ & $\%$ & $\mathrm{~N}$ & $\%$ & \begin{tabular}{|l}
$\mathrm{N}$ \\
\end{tabular} & $\%$ & $\mathrm{~N}$ & $\%$ & $\mathrm{~N}$ & $\%$ & $\mathrm{~N}$ & $\%$ & $\mathrm{~N}$ & $\%$ \\
\hline NVD & 5 & 16.7 & 17 & 56.7 & 10 & 33.3 & 22 & 73.3 & 3 & 10.0 & 21 & 70.0 & 9 & 30.0 & 8 & 26.7 \\
\hline $\mathrm{CS}$ & 25 & 83.3 & 13 & 43.3 & 20 & $\begin{array}{ll}66.7 \\
\end{array}$ & 8 & 26.7 & 27 & 90.0 & 9 & 30.0 & 21 & 70.0 & 22 & 73.3 \\
\hline p-value & $P-1$ & te & If & $=0$. & 00 & $\mathrm{t}=6$ & & P-va & te & ter) $=$ & 0.0 & & $t=$ & & $\frac{1}{16.5}$ & \\
\hline
\end{tabular}

${ }^{1}$ Motivational Interviewing group; ${ }^{2}$ Information, Motivation and Behavior group; ${ }^{3}$ Information, Motivation and Behavior through mobile application group

The number of women undergone CS decreased in the intervention groups after the interventions ( $\mathrm{P}$-value $=0.012$ ); and this decrease occurred more among women in the IMB-App group than the other intervention groups (Table 5).

Table 5: Mode of delivery among intervention and control groups $(n=120)$

\begin{tabular}{|l|l|l|l|l|}
\hline \multirow{3}{*}{ Study groups (n) } & \multicolumn{4}{|l|}{ Mode of delivery } \\
\cline { 2 - 5 } & \multicolumn{2}{|l|}{ NVD $^{1}$} & \multicolumn{2}{l|}{ CS $^{2}$} \\
\cline { 2 - 5 } & $\mathrm{N}$ & $\%$ & $\mathrm{~N}$ & $\%$ \\
\hline MI (30) & 13 & 43.3 & 17 & 56.7 \\
\hline IMB (30) & 10 & 33.3 & 20 & 66.7 \\
\hline IMB-APP (30) & 17 & 56.7 & 13 & 43.3 \\
\hline Control (30) & 5 & 16.7 & 25 & 83.3 \\
\hline Total (120) & 45 & 37.5 & 75 & 62.5 \\
\hline $\mathbf{X}^{\mathbf{2}}$ & \multicolumn{5}{|l|}{ p.value $=\mathbf{0 . 0 1 2}$} \\
\hline
\end{tabular}

${ }^{1}$ Normal Vaginal Delivery; 2 Cesarean Section

Table 6 compares women's intention on mode of delivery after the intervention with women' mode of delivery. Although $56.7 \%$ women preferred NVD, only $37.5 \%$ of them underwent NVD and $62.5 \%$ underwent CS.

Table 6: Comparison between women's intention on mode of delivery after the intervention and mode of delivery

\begin{tabular}{|c|c|c|c|c|}
\hline \multirow{2}{*}{ Variable } & Women's intention on mode of delivery after the intervention & \multicolumn{2}{|c|}{ Delivery mode } \\
\cline { 2 - 5 } & $\mathrm{N}$ & $\%$ & $\mathrm{~N}$ & $\%$ \\
\hline NVD & 68 & 56.7 & 45 & 37.5 \\
\hline CS & 52 & 43.3 & 75 & 62.5 \\
\hline
\end{tabular}

\section{Discussion}

The purpose of this study was to examine the effect of "motivational interviewing" and "information, motivation and behavioral skills" on choosing mode of delivery in pregnant women. Findings indicated that these psycho-education interventions had positive effects on decreasing CS rates in pregnant women. Our study also showed that the interventions based on psych-education contributed to improvements in women's self-efficacy towards choosing mode of delivery and their intention.

World Health Organization recommends educational intervention to reduce unnecessary CS. Four types of interventions have been proposed by WHO: (1) Childbirth training workshops; (2) Nurse-led applied relaxation training programme; (3) Psychosocial couple-based prevention programme; and (4) Psychoeducation (for women with fear of pain; comprising information about fear and anxiety, fear of childbirth, normalization of individual reactions, stages of labour, hospital routines, birth process, and pain relief [led by a therapist and midwife], among other topics). However, high-quality evidence on educational intervention is lacking systematically; and these interventions are recommended to reduce caesarean births only with targeted monitoring and evaluation which reflect the lack of strong evidence Psycho-education interventions have been recommended as useful approaches to decrease the increasing trend of CS (37). 
Due to the situation of our target group and probable issues with long-term interventions, we used brief interventions that were tailored with their prenatal care clinic appointments. The effectiveness of brief interventions has been approved by several studies $(61,62)$. A Cochrane systematic review conducted by Chen et al (40) in China on non-clinical interventions to reduce unnecessary CS showed that psych-education interventions were effective in reducing unnecessary CS.

Our study has shown that the psycho-education interventions based on IMB and MI were effective in reducing the rate of CS. Our interventions also decreased women's intention to undergo CS significantly after the interventions (before childbirth). However, the rate of real NVDs was not equal to the reported ones as the intentions of women. It shows the role of other possible significant factors forcing women to undergo CS, such as doctors' recommendations at childbirth. This highlights the need to implement multidimensional interventions to decrease the rate of CS in Iran

According to the results of this study, self-efficacy of pregnant women improved after the interventions. Self-efficacy can help women and improve their ability to cope with labor; and women who have high self-efficacy could cope with labor pain during childbirth much better than the others. Our study showed that women with higher self-efficacy, chose more NVDs that is consistent with the results of Dilks et al (63) and Taheri et al (58). Taheri et al reported that implementing a curriculum-based strategy to increase self-efficacy in pregnant women was effective in encouraging women to choose NVD (58). Thus, it seems that improvements in self-efficacy could empower women to choose proper mode of delivery.

Our study showed that mobile application intervention group had higher improvements in self-efficacy and intention to choose mode of delivery than other intervention groups. Educational interventions at any time and in any place can easily provide persistent educational content to anyone who needs it. The mobile application interventions can be used in a wide range in Iran; because a wide range of people now in Iran uses cellphones. According to reports, the number of mobile cellular subscriptions in Iran, with a population of 80 million, has increased to 118 million in 2019; and women make about 45 percent of cellphone users (64). Motivational messages, management tools, and behavior change messages can be widely available to individuals via mobile phones (53). The results are in agreement with the results of previous studies that support the effectiveness of mobile technology to influence lifestyles and provide health education and behavior change contents $(54,55,65)$. The value of using $\mathrm{m}$-health has been highlighted these months with COVID-19 pandemic. Pregnant women as at risk group need to be careful about social contacts and m-health provides them appropriate educational interventions through proper channel.

\section{Implications for policymakers}

Our findings showed that psych-education may be effective approach as a policy option for tackling the outcomes of unnecessary CS. The results of this study can draw policy attention appropriately to implement this type of interventions for the larger health programmes in local, national, and/or regional level. Now that smart mobile phones are accessible for everyone around the country, using mobile application also makes it possible to determine the implications of this diverse intervention for policymaking based on the situations. Using these simple, non-expensive, tailored to women's prenatal care appointments, culture-oriented brief interventions might fruitfully be used to help women make proper and self-efficient decisions regarding their mode of delivery. However, wide variety of implementation strategies should be considered to deliver this kind of brief intervention in a wide population and through prenatal clinics.

\section{Implications for further studies}

Implementation frameworks related to the psycho-education interventions should be studied to identify barriers and facilitators of implementing such strategies. We have conducted our study in a private hospital. Future studies should assess the effectiveness of the interventions in public sector among a larger number of participants measuring more outcomes such as APGAR score of newborn babies. Conducting studies to implement multifaceted strategies targeting at women, health professionals, and healthcare systems is suggested. In order to conduct such interventions, the reasons behind the high rates of $\mathrm{CS}$ in the context should be identified to design proper psycho-education interventions.

\section{Strengths and limitations}

This was a non-clinical interventional study targeted at women that helped in decreasing unnecessary CS among participant women. We used a robust randomized controlled trial design and found effective results. However, the study was conducted in a private hospital with limited number of participants. We ensured women that their responses would not affect the service they would receive and ensured confidentiality. We used the results of two other studies conducted among Iranian women $(19,35)$ to design the content of the intervention as a culture-oriented content. Moreover, we tailored the brief interventions to women's prenatal care appointments.

\section{Conclusion}

Our study showed that that brief pchyo-education intervention based on IM and IMB were effective to improve self-efficacy and intention among pregnant women and helped them to choose their proper mode of delivery. Moreover, the study showed that implementing the psycho-education intervention through mobile application was more effective in reducing CS rate and improving self-efficacy and behavior intention than the others two intervention groups. Using these psycho-education strategies targeted at women along with interventions targeted at healthcare providers and health system would probably be effective in reducing unnecessary CS rate in local and/or regional levels.

\section{Abbreviations}

CS= Cesarean Section

NVD= Normal Vaginal Delivery 
WHO= World Health Organization

MI=Motivational Interviewing

IMB = Information Motivation and Behavioural skills model

IMB-App= Information, Motivation and Behavioral skill model through mobile application

\section{Declarations}

\section{Acknowledgements}

We are grateful to the women who participated in our study.

\section{Data sharing}

Additional documents related to this study are available on request to the corresponding author. The trial protocol and the pictures of the Mobile-Application are also available in the appendix.

\section{Funding}

This study has been financially supported by Tehran University of Medical Sciences (TUMS) and Iran National Science Foundation (INSF). The roles of the funders were to monitor the corresponding research planning and progression.

\section{Authors' contributions}

E. Sh., M. Sh., and M. A. conceptualized the study. E. Sh. M. Sh., and H. P. developed the study design and data collection plans. M. Sh. collected the data. M. Sh. and A. R. F. analyzed the data. E. Sh. and M. Sh. drafted the manuscript. All authors provided critical inputs on an earlier version of the manuscript and read and approved the final version of the manuscript.

\section{Ethics approval and consent to participate}

This was conducted as part of a PhD thesis project at Tehran University of Medical Sciences (TUMS). This study was approved by the School of Public Health, TUMS Ethics Committee (Ethics code: IR.TUMS.SPH.REC-1397-130). All participants were informed about the study and purposes and were ensured that all information collected would remain confidential. Every participant signed an informed consent form.

\section{Consent for publication}

The relevant written and informed consent for publication was obtained from the participants at recruitment.

\section{Competing interests}

The authors declare that they have no competing interests.

\section{References}

1. Ahmad Nia S, Delavar B, Eini Zinab H, Kazemipour S, Mehryar A, Naghavi M. Caesarean section in the Islamic Republic of Iran: prevalence and some sociodemographic correlates. 2009.

2. Creasy R, Resnik R, lams J. Clinical aspects of normal and abnormal labor. Maternal-Fetal Medicine. 1984;5:543-9.

3. Guise J-M, Eden K, Emeis C, Denman MA, Marshall N, Fu RR, et al. Vaginal birth after cesarean: new insights. Evidence report/technology assessment. 2010(191):1.

4. Menacker F, Hamilton BE. Recent trends in cesarean delivery in the United States. 2010.

5. Sandall J, Tribe RM, Avery L, Mola G, Visser GH, Homer CS, et al. Short-term and long-term effects of caesarean section on the health of women and children. The Lancet. 2018;392(10155):1349-57.

6. WHO. WHO Statement on Caesarean Section Rates. World Health Organization WHO; 2018. p. 12.

7. Betrán AP, Ye J, Moller A-B, Zhang J, Gülmezoglu AM, Torloni MR. The increasing trend in caesarean section rates: global, regional and national estimates: 1990-2014. PloS one. 2016;11(2):e0148343.

8. Yavangi M, Sohrabi M-R, Alishahi Tabriz A. Effect of Iranian ministry of health protocols on cesarean section rate: a quasi-experimental study. Journal of research in health sciences. 2013;13(1):48-52.

9. Azami-Aghdash S, Ghojazadeh M, Dehdilani N, Mohammadi M. Prevalence and causes of cesarean section in Iran: systematic review and meta-analysis. Iranian journal of public health. 2014;43(5):545.

10. Omani-Samani R, Mohammadi M, Almasi-Hashiani A, Maroufizadeh S. Cesarean section and socioeconomic status in Tehran, Iran. Journal of research in health sciences. 2017;17(4) 
11. mohamadbeigi a, tabatabaee sh, mohammad salehi $n$, yazdani m. Factors Influencing Cesarean Delivery Method in Shiraz Hospitals. Iran Journal of Nursing. 2009;21(56):37-45.

12. Betrán AP, Temmerman M, Kingdon C, Mohiddin A, Opiyo N, Torloni MR, et al. Interventions to reduce unnecessary caesarean sections in healthy women and babies. The Lancet. 2018;392(10155):1358-68.

13. Gregory KD, Jackson S, Korst L, Fridman M. Cesarean versus vaginal delivery: whose risks? Whose benefits? American journal of perinatology. 2012;29(01):07-18.

14. Souza JP, Gülmezoglu A, Lumbiganon P, Laopaiboon M, Carroli G, Fawole B, et al. Caesarean section without medical indications is associated with an increased risk of adverse short-term maternal outcomes: the 2004-2008 WHO Global Survey on Maternal and Perinatal Health. BMC medicine. 2010;8(1):71.

15. Organization WH. WHO Statement on Caesarean Section Rates. 2018. p. 12.

16. Ramashwar S. In Asia, cesarean section associated with increased risk of neonatal mortality. International Perspectives on sexual and reproductive health. 2010;36(2):116.

17. MacFarlane AJ, Blondel B, Mohangoo A, Cuttini M, Nijhuis J, Novak Z, et al. Wide differences in mode of delivery within Europe: risk-stratified analyses of aggregated routine data from the Euro-Peristat study. BJOG: An International Journal of Obstetrics \& Gynaecology. 2016;123(4):559-68.

18. Zwecker P, Azoulay L, Abenhaim HA. Effect of fear of litigation on obstetric care: a nationwide analysis on obstetric practice. American journal of perinatology. 2011;28(04):277-84.

19. Mahboube Shirzad ES, Khadijeh Hajimiri, Ana Pilar Betran, Shayeste Jahanfar, Meghan A. Bohren, Newton Opyo, Qian Long, Carol Kingdon, Mercedes Colomar, Mehrandokht Abedini. Prevalence of and reasons for women's, family members', and health professionals' preferences for cesarean section in Iran: A mixed-methods systematic review. 2019.

20. ABBASPOUR Z, MOGHADDAM BL, AHMADI F, KAZEMNEJAD A. Women's fear of childbirth and its impact on selection of birth method: a qualitative study. 2014.

21. Bayrami R, Valizadeh L, Zaheri F. Nulliparous women's childbirth experiences: A phenomenological Study. 2011.

22. Latifnejad-Roudsari R, Zakerihamidi M, Merghati-Khoei E, Kazemnejad A. Cultural perceptions and preferences of Iranian women regarding cesarean delivery. Iranian journal of nursing and midwifery research. 2014;19(7 Suppl1):S28.

23. Shams M, Mousavizadeh A, Parhizkar S, Maleki M, Angha P. Development a tailored intervention to promote normal vaginal delivery among primigravida women: a formative research. The Iranian Journal of Obstetrics, Gynecology and Infertility. 2016;19(30):9-25.

24. Hajian S, Shariati M, Najmabadi KM, Yunesian M, Ajami ME. Psychological predictors of intention to deliver vaginally through the extended parallel process model: a mixed-method approach in pregnant Iranian women. Oman medical journal. 2013;28(6):395.

25. Borghei NS, Taghipour A, Latifnejad Roudsari R. The concern of fetal health: women's experiences of worries during pregnancy. The Iranian Journal of Obstetrics, Gynecology and Infertility. 2016;19(28):10-21.

26. Yazdizadeh B, Nedjat S, Mohammad K, Rashidian A, Changizi N, Majdzadeh R. Cesarean section rate in Iran, multidimensional approaches for behavioral change of providers: a qualitative study. BMC health services research. 2011;11(1):159.

27. Sanavi FS, Rakhshani F, Ansari-Moghaddam A, Edalatian M. Reasons for Elective Cesarean Section amongst Pregnant Women; A Qualitative Study. Journal of reproduction \& infertility. 2012;13(4):237-40.

28. Shahoei R, Rostami F, Khosravi F, Ranayi F, Hasheminasab L, Hesami K, et al. Women Lived Experience of Choice of Cesarean Delivery: A Phenomenology Study. The Iranian Journal of Obstetrics, Gynecology and Infertility. 2014;17(104):1-10.

29. Latifnejad Roudsari R, Zakerihamidi M, Merghati Khoei E. Socio-Cultural Beliefs, Values and Traditions Regarding Women's Preferred Mode of Birth in the North of Iran. International journal of community based nursing and midwifery. 2015;3(3):165-76.

30. Mobarakabadi SS, Najmabadi KM, Tabatabaie MG. Ambivalence towards childbirth in a medicalized context: a qualitative inquiry among Iranian mothers. Iranian Red Crescent Medical Journal. 2015;17(3).

31. Besharati F, Hazavehei S, Moeini B, Moghimbeigi A. Effect of educational interventions based on theory of planned behavior (TPB) in selecting delivery mode among pregnant women referred to Rasht health centers. Journal of Zanjan University of Medical Sciences and Health Services. 2011;19(77):10.

32. Sharifirad G, Rezaeian M, Soltani R, Javaheri S, Mazaheri MA. A survey on the effects of husbands' education of pregnant women on knowledge, attitude, and reducing elective cesarean section. Journal of education and health promotion. 2013;2.

33. Soltani F, Eskandari Z, Khodakarami B, Parsa P, Roshanaei G. The Effect of Self-Efficacy Oriented Counselling on Controlling the Fear of Natural Delivery in Primigravida Women. Journal of Pharmaceutical Sciences and Research. 2017;9(10):1757-61.

34. Rasouli M, Mousavi SA, Khosravi A, Keramat A, Fooladi E, Atashsokhan G. The impact of motivational interviewing on behavior stages of nulliparous pregnant women preparing for childbirth: a randomized clinical trial. Journal of Psychosomatic Obstetrics \& Gynecology. 2018;39(3):237-45.

35. Shirzad M, Shakibazadeh E, Betran AP, Bohren MA, Abedini M. Women's perspectives on health facility and system levels factors influencing mode of delivery in Tehran: a qualitative study. Reproductive health. 2019;16(1):15.

36. Rashidian A, Moradi G, Takian A, Sakha MA, Salavati S, Faraji O, et al. Effects of the Health Transformation Plan on caesarean section rate in the Islamic Republic of Iran: an interrupted time series. 2018.

37. Organization WH. WHO recommendations non-clinical interventions to reduce unnecessary caesarean sections: World Health Organization; 2018.

38. Walker R, Turnbull D, Wilkinson C. Strategies to address global cesarean section rates: a review of the evidence. Birth. 2002;29(1):28-39. 
39. Kingdon C, Downe S, Betran AP. Women's and communities' views of targeted educational interventions to reduce unnecessary caesarean section: a qualitative evidence synthesis. Reproductive health. 2018;15(1):130.

40. Chen I, Opiyo N, Tavender E, Mortazhejri S, Rader T, Petkovic J, et al. Non-clinical interventions for reducing unnecessary caesarean section. Cochrane Database of Systematic Reviews. 2018(9).

41. Bastani F, Hidarnia A, Montgomery KS, Aguilar-Vafaei ME, Kazemnejad A. Does relaxation education in anxious primigravid Iranian women influence adverse pregnancy outcomes?: a randomized controlled trial. The Journal of perinatal \& neonatal nursing. 2006;20(2):138-46.

42. Saisto T, Salmela-Aro K, Nurmi J-E, Könönen T, Halmesmäki E. A randomized controlled trial of intervention in fear of childbirth. Obstetrics \& Gynecology. 2001;98(5):820-6.

43. Rouhe H, Salmela-Aro K, Toivanen R, Tokola M, Halmesmäki E, Saisto T. Obstetric outcome after intervention for severe fear of childbirth in nulliparous women-randomised trial. BJOG: An International Journal of Obstetrics \& Gynaecology. 2013;120(1):75-84.

44. Navaee M, Abedian Z. Effect of role play education on primiparous women's fear of natural delivery and their decision on the mode of delivery. Iranian journal of nursing and midwifery research. 2015;20(1):40.

45. Miller WR. Motivational interviewing: research, practice, and puzzles. Addictive behaviors. 1996;21(6):835-42.

46. Hoseini Haji SZ, Firoozi M, Asghari Pour N, Taghi Shakeri M. Impact of Motivational Interviewing on Women's Knowledge, Attitude and Intention to Choose Vaginal Birth after Caesarean Section: A Randomized Clinical Trial. Journal of Midwifery and Reproductive Health. 2019:1-11.

47. Fisher WA, Fisher JD, Harman J. The information-motivation-behavioral skills model: A general social psychological approach to understanding and promoting health behavior. Social psychological foundations of health and illness. 2003;82:106.

48. Singh S. Study of the effect of information, motivation and behavioural skills (IMB) intervention in changing AIDS risk behaviour in female university students. AIDS care. 2003;15(1):71-6.

49. Ndebele M, Kasese-Hara M, Greyling M. Application of the information, motivation and behavioural skills model for targeting HIV risk behaviour amongst adolescent learners in South Africa. SAHARA-J: Journal of social aspects of HIV/AIDS. 2012;9(4):37-47.

50. Bryan AD, Fisher JD, Benziger TJ. HIV prevention information, motivation, behavioural skills and behaviour among truck drivers in Chennai, India. AiDS. 2000;14(6):756-8.

51. Kalichman S, Stein J, Malow R, Averhart C, Devieux J, Jennings T, et al. Predicting protected sexual behaviour using the Information-Motivation-Behaviour skills model among adolescent substance abusers in court-ordered treatment. Psychology, Health \& Medicine. 2002;7(3):327-38.

52. Islam R, Islam R, Mazumder T. Mobile application and its global impact. International Journal of Engineering \& Technology (IJEST). 2010;10(6):72-8.

53. Free C, Phillips G, Galli L, Watson L, Felix L, Edwards P, et al. The effectiveness of mobile-health technology-based health behaviour change or disease management interventions for health care consumers: a systematic review. PLoS medicine. 2013;10(1):e1001362.

54. Whittaker R, McRobbie H, Bullen C, Rodgers A, Gu Y. Mobile phone-based interventions for smoking cessation. Cochrane Database of Systematic Reviews. 2016(4).

55. Dayer L, Heldenbrand S, Anderson P, Gubbins PO, Martin BC. Smartphone medication adherence apps: potential benefits to patients and providers. Journal of the American Pharmacists Association. 2013;53(2):172-81.

56. Price M, Yuen EK, Goetter EM, Herbert JD, Forman EM, Acierno R, et al. mHealth: a mechanism to deliver more accessible, more effective mental health care. Clinical psychology \& psychotherapy. 2014;21(5):427-36.

57. Shakibazadeh E, Shirzad M, Foroushani A, Abedini M, Poursharifi H, Babaei S. Effect of" motivational interviewing" and" information, motivation and behavioral skills" on choosing the mode of delivery in pregnant women: A study protocol for a randomized controlled trial. 2020.

58. Taheri Z, Mazaheri MA, Khorsandi M, Hassanzadeh A, Amiri M. Effect of educational intervention on self-efficacy for choosing delivery method among pregnant women in 2013. International journal of preventive medicine. 2014;5(10):1247.

59. Khorsandi M, Ghofranipour F, Faghihzadeh S, Hidarnia A, Akbarzadeh Bagheban A, Aguilar-Vafaie ME. Iranian version of childbirth self-efficacy inventory. Journal of Clinical Nursing. 2008;17(21):2846-55.

60. ShahrakiSanavi F, Navidian A, Rakhshani F, Ansari-Moghaddam A. The effect of education on base the Theory of Planned Behavior toward normal delivery in pregnant women with intention elective cesarean. Hormozgan Medical Journal. 2014;17(6):531-9.

61. Walton GM, Cohen GL. A brief social-belonging intervention improves academic and health outcomes of minority students. Science. 2011;331(6023):1447-51.

62. Borsari B, Carey KB. Effects of a brief motivational intervention with college student drinkers. Journal of consulting and clinical psychology. 2000;68(4):728.

63. Dilks FM, Beal JA. Role of self-efficacy in birth choice. The Journal of perinatal \& neonatal nursing. 1997;11(1):1-9.

64. I.C.T Mo. Iranian, Foreign Officials to Attend Seminar on "Woman's Empowerment through ICT" 2020 [Available from: https://www.ict.gov.ir/en/aboutus.

65. Ashoorkhani M, Bozorgi A, Majdzadeh R, Hosseini H, Yoonessi A, Ramezankhani A, et al. Comparing the effectiveness of the BPMAP (Blood Pressure Management Application) and usual care in self-management of primary hypertension and adherence to treatment in patients aged 30-60 years: Study protocol for a randomized controlled trial. Trials. 2016;17(1):511.

\section{Figures}




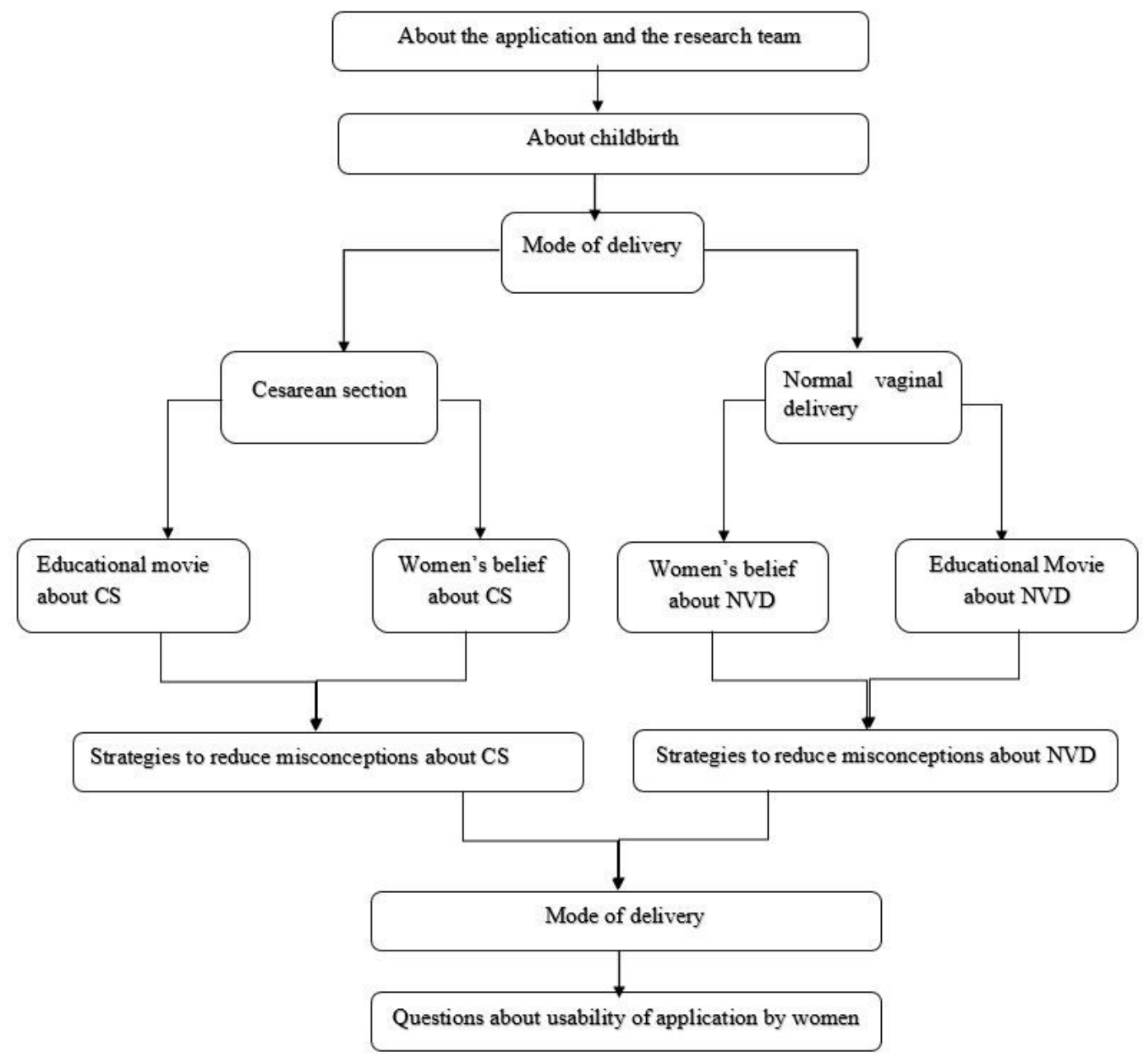

Figure 1

Flowchart 1: process of designing application

\section{Supplementary Files}

This is a list of supplementary files associated with this preprint. Click to download.

- additionalfile1.doc

- supplementaryfile1.docx 